\title{
Correction to: LncRNA GAS5 activates the HIF1A/VEGF pathway by binding to TAF15 to promote wound healing in diabetic foot ulcers
} Wei-Xia Peng $\cdot$ Pei-Xiang He $\cdot$ Li-Jun Liu $\cdot$ Ting Zhu $\cdot$ Ya-Qin Zhong $\cdot$ Lin Xiang $\cdot$ Ke Peng $\cdot$ Jing-Jin Yang $\cdot$
Guang-Da Xiang

Published online: 5 May 2021

(c) The Author(s), under exclusive licence to United States and Canadian Academy of Pathology 2021

Correction to: Laboratory Investigation

https://doi.org/10.1038/s41374-021-00598-2

The original version of this article unfortunately contained a mistake. Due to a typeset error the Summary was omitted. We apologize for the error. The original article has been corrected.

\author{
Summary \\ Long noncoding RNAs GAS5 activates the HIF1A/VEGF \\ pathway by binding to TAF15 (a component of RNA \\ polymerase II), resulting in accelerated wound healing in \\ diabetic foot ulcers. These findings may provide a theore- \\ tical basis for clinical treatment.
}

